\title{
Aloe vera for Prevention of Acute Radiation Proctitis in Colorectal Cancer: A Preliminary Randomized, Placebo-Controlled Clinical Trial
}

Ebrahim Salehifar ( $D$ Esalehifar52@gmail.com)

Mazandaran University of Medical Sciences

adeleh sahebnasagh

North Khorasan University of Medical Sciences

fatemeh saghafi

Shahid Sadoughi University of Medical Sciences and Health Services Yazd Research and Clinical Centre for Infertility

abbas alipour

Mazandaran University of Medical Sciences

Arash Ghasemi

Mazandaran University of Medical Sciences

Jafar Akbari

Mazandaran University of Medical Sciences

Mohammadreza Safdari

North Khorasan University of Medical Sciences

Hassan Rezai Ghaleno

Lorestan University of Medical Sciences

Research article

Keywords: Acute proctitis, Aloe vera, Colorectal Cancer, Radiotherapy, Topical Formulation

Posted Date: April 25th, 2020

DOI: https://doi.org/10.21203/rs.3.rs-22563/v1

License: (c) (i) This work is licensed under a Creative Commons Attribution 4.0 International License.

Read Full License 


\section{Abstract}

Background Acute radiation proctitis (ARP) is a common complication of radiation therapy (RT). This adverse effect in severe cases ultimately results in interruption of RT and poor outcome. Aloe vera contains multiple antioxidant and anti-inflammatory compounds This study was aimed to evaluate the preventive effects of Aloe vera in ARP in colorectal cancer.

Method: twenty eligible colorectal cancer patients, who received radiation, were randomized to receive Aloe vera $3 \%$ ointment or placebo for six weeks. At each weekly visit, ARP symptoms were evaluated by Radiation Therapy Oncology Group (RTOG) and clinical presentation criteria. The lifestyle impact of the symptoms, depression and anxiety for assessment of psychosocial status of patients, and quantitative CRP were evaluated as secondary endpoints.

Results there was a significant improvement in the symptom index for diarrhea and total RTOG scores and quantitative CRP levels (between-subject differences or group effect) $(p<0.05)$ in Aloe group. The overall time trend (within-subject differences or time effect) was statistically significant for rectal bleeding, rectal pain, total clinical scores, proctitis, cystitis, and total RTOG $(P<0.05)$ in Aloe vera group. The median lifestyle score improved significantly with Aloe vera. Intervention patients had a lower probability of presenting anxiety in their overall HAD scale during RT.

Conclusion The use of topical formulation of Aloe vera $3 \%$ diminished the severity of ARP in colorectal cancer patients. Trial registration: Current Controlled Trials IRCT201606042027N6, registrations date: 2016-04-09.

\section{Background}

Aloe vera is an herbal remedy with a well-established history for its therapeutic properties in various disorders. It is well-known for its anti-oxidant, anti-inflammatory, analgesic, antiproliferative, anticarcinogenic and antiaging properties. This plant has been also used in radiation-induced skin reactions and proctitis treatment for its anti-inflammatory, anti-oxidant and analgesic properties in previous studies $(1,2)$. Our understanding of the mechanism underlying the action of Aloe vera in healing wounds is still not clear, but some suggest that it plays its role through suppression of cyclooxygenase-2 (3).

Acute radiation proctitis (ARP) is a common complication of pelvic radiotherapy (RT) to malignant diseases of lower abdomen and pelvis. ARP presents as rectal bleeding and pain, diarrhea, and fecal urgency, with negative impact on quality of life (4-6). The molecular mechanism of ARP is due to an enhanced production of free oxygen species (4).

Colorectal cancer is one of the leading causes of death worldwide which has a survival rate $<50 \%$ after 5 years (7). Interestingly, the most common symptoms associated with colon cancer is similar to radiation proctitis and as followed: abdominal pain (44\%), change in bowel habits (43\%), hematochezia 
or melena (40\%) (8). Preoperative RT is used as an adjuvant modality for rectal cancer and a systematic review of 28 clinical trials have shown its effectiveness in reducing risk of local recurrence and death from rectal cancer (9). Therefore, local treatment by means of pelvic RT may cause subsequent anorectal injury which may have a great deal of overlap between the symptoms of cancer and ARP (10).

Different modalities for prevention of ARP are available. Much effort has been made to shield the rectum during radiation, but it is generally inapplicable or hard (11). Thereby, the risk of damage to the rectum is high. Other less available modalities include precise dose-planning, and new radiation techniques, such as the use of contemporary conformal radiation therapy techniques (eg, intensity modulated radiation therapy, intensity guided radiation therapy) which minimize the dose of radiation to the rectum while maximizing dose to the tumor $(12,13)$. Medical therapy (eg, amifostine) has only a minimal effect and are not widely used $(14,15)$.

Evaluation of Aloe vera in a randomized trial against placebo in patients suffering from colorectal cancer is debatable, since the best current practice for prevention of ARP is not known to rely on. Specially in this population, scientific studies remain infrequent and rare. Considering the molecular mechanism of Aloe vera, the underlying pathophysiology of ARP, disturbing symptoms associated with colorectal cancer, and also the uncertainties about a widely accepted prophylactic treatment for radiation proctitis, we decided to examine the issue in a preliminary prospective randomized, double-blind, placebo controlled clinical trial in patients undergoing RT to the pelvis.

\section{Methods}

Over a period of 12 months, 20 patients who met the inclusion criteria for the study were consecutively allocated to intervention or control group at a university affiliated radiation oncology department. The eligible participants had been evaluated for preventing effects of Aloe vera rectal ointment in ARP for colorectal cancer. The trial profile is shown in Fig. 1. Patients were interviewed and given written informed consent. Those individual aged 18 years or more who were undergoing radiation for colorectal cancer were considered for this trial. They must not have had evidence of active infection; evidence of other sources of hematochezia including inflammatory bowel disease, and hemorrhoids; anal incontinence, anorectal fistula, anorectal stenosis; pregnancy or breast feeding, female of child-bearing age not taking adequate contraception; known allergy to any ingredients of the ointment; and concomitant use of antibiotics or steroids.

For preparation and formulation of Aloe vera ointment, pure spray-dried Aloe vera powder was applied (Giah salamat nasim faraz, Fars, Iran). The inner gel of the plant was formulated in the ointment base (2).

The patients were randomized in a double-blind manner to receive either an Aloe vera $3 \%$ ointment or placebo. The study medication (Aloe vera or placebo) was to be applied rectally with an applicator. The dose used was $1 \mathrm{~g}$ twice a day starting from the first day of radiation initiation and then during six weeks of radiotherapy. The patients were assessed by completing questionnaires at study initiation and weekly until 6 weeks following initiation of radiation therapy. 
The ointment tubes were exactly indistinguishable. Patients, clinician and the investigator were not aware of intervention or placebo arms. Trial allocation was according to Random Number Table, using permuted randomization blocks of four. Compliance assessment was determined by asking the participants to bring their tubes for weekly visits. At each weekly visit, ARP symptoms were evaluated by Radiation Therapy Oncology Group (RTOG) and clinical presentation criteria (2). Four signs of rectal pain and bleeding, bowel movement and fecal urgency were reported. For each sign, a scale from 0 (not present) to 4 (most symptomatic) was used. The maximum overall score was $16(2,4)$. The primary outcome measures were prevention of proctitis development (defined as lower scores of RTOG Criteria and Radiation Proctitis Symptom Score). All the scores were assessed by the same investigator of clinical responses, blind to the treatment given.

In addition to our primary end point, we evaluated secondary end points of quality of life (QOL), psychosocial status using Hospital Anxiety-Depression Scale (HAD), and also changes in laboratory measures of inflammation [quantitative measurement of $\mathrm{C}$ - reactive protein (CRP)], hemoglobin, and platelet count. Possible adverse effects of the trial medications were also recorded.

Quantitative CRP was measured by obtaining serum samples at baseline, week 2, and week 6. CRP-Latex Bionik slide agglutination kit with a specificity of $95.6 \%$ and specificity of $96.2 \%$ was applied.

Psychosocial status of patients was assessed by HAD Scale. At each visit, patients were requested to fill out quality of life and HAD form by themselves.

For statistical analyses, we used the Shapiro-Wilk test to test whether data were normally distributed. Descriptive baseline characteristics for two groups (Aloe ointment and placebo ointment) comparisons were tabulated as mean $\pm S D$, median (inter-quartile range) or as percentages. Comparing between two groups for categorical data were statistically analyzed using chi- square or Fisher-exact test and for continuous data were statistically analyzed using t-test and Mann-Whitney $U$ test. The primary efficacy was examined using intention-to-treat analysis. We used a generalized estimating equation (GEE) model to estimate the differences in values of endpoints at each time point between the two groups (between group effects). Within group effects were assessed with the Friedman test. A $p$-value of 0.05 or less was considered statistically significant. Data were analyzed using IBM SPSS statistics version 16 and stata version 10 .

\section{Results}

Over a period of 12 months, 20 patients completed a cycle of external-beam radiation therapy to the pelvis. They were randomly assigned to receive Aloe ointment $(n=12)$ or placebo ointment $(n=8)$. Demographic and baseline clinical characteristics of enrolled patients were presented in table 1 . All patients were followed-up regularly. However, two patients (one on each arm) withdrew the study, because of elective discontinuation of RT. Average patient age was 55.3 years, with no significant difference between groups. The rectal ointments were well tolerated in all patients. No patient in either group experienced any adverse effects. Our patients were objectively monitored with a patient diary. Compliance 
was good as assessed by comparing the actual and estimated volumes of residual ointment. Both intervention and placebo groups were well balanced with respect to baseline patient characteristics including gender ratio, body mass index, dose of radiotherapy in each session, concomitant medication and comorbidities (Table 1). Flow diagram of the study population selection is displayed in figure 1.

\section{Symptom Scores}

\section{Effect of Aloe vera rectal ointment on symptoms}

The effect of Aloe vera rectal ointment on prevention of individual proctitis symptoms during the course of radiation treatment, are described, as median (Q25-Q75) of primary endpoints, in table 2 in detail. Figure 2 and 3 show outcomes by applying Aloe or placebo ointments over six weeks of the study. As shown in table 2 , the scores getting decreased and there was a statistically significant time trend (withinsubject differences or time effect) for rectal bleeding, rectal pain, total clinical scores, proctitis, cystitis, and total RTOG $(\mathrm{P}<0.05)$ in Aloe vera group.

Diarrhea and RTOG total scores in Aloe vera group was better than placebo group and there were statistically significant differences between groups (between-subject differences or group effect) $(\mathrm{p}<0.05)$. As a matter of fact, with exception of diarrhea and RTOG total scores, all measures of clinical toxicity did not achieve a statistical significant difference with Aloe vera ointment, compared to the placebo arm. However, the preventive effect of intervention group, in comparison to the placebo, still favored Aloe group. As illustrated, the average scores in placebo arm for rectal pain and diarrhea followed an upward trend during 6 weeks of study.

\section{Secondary end points}

QOL, depression and anxiety for assessment of psychosocial status of patients, and quantitative CRP were evaluated as secondary endpoints.

As shown in table 2, the median lifestyle score improved significantly with Aloe vera, (within-subject differences or time effect) $(P<0.05)$, whereas the overall score value increased significantly in placebo group, indicating the worsening quality of life for this arm of the study during the study period. Furthermore, anxiety scores in HAD scale during RT getting decreased and there was a statistically significant time trend (within-subject differences or time effect) $(P<0.05)$ in Aloe vera ointment group. Quantitative measurement of CRP score getting decreased (Fig 4). CRP scores in Aloe vera group was better than placebo group and there were statistically significant differences between groups (betweensubject differences or group effect) $(p<0.05)$.

\section{Discussion}

The present study is the first randomized, double-blind, placebo-controlled clinical trial evaluating the effectiveness of topical Aloe vera $3 \%$ ointment at a dose of $1 \mathrm{~g} /$ twice a day in the prevention of ARP in 
colorectal cancer patients receiving RT. Results from this study show that Aloe vera gel is superior to the placebo in the prevention of the incidence of ARP and related symptoms in some aspects.

Numerous risk factors play an important role in radiation-induced skin damage. Continuous free radical production potentiates the intracellular inflammatory response. The main mechanism of RT-induced proctitis is DNA damage and cell death which trigger the inflammatory responses following exposure to radiation (16). Previous studies indicated that anti-inflammatory agents can decrease the severity of ARP partly by reducing inflammation $(17,18)$.

It is interesting to note that radiation-induced-mucositis has a similar pathophysiology to that of ARP. In both conditions, oxygen species probably have a significant influence on initiation and progression of inflammatory lesions. Previous studies have demonstrated the healing effects of Aloe vera in radiationinduced oral mucositis by scavenges free radicals. Although the preventive effects of Aloe vera in ARP has not been investigated earlier, this is giving us a clue regarding the beneficial effects of this herbal medicine (18). Different modalities for ARP prevention, including shielding the rectum during radiation, precise dose-planning, new radiation techniques, and medical therapy (i.e., amifostine) have indicated some degrees of improvements in several clinical trials. Clinically significant results with reduction of ARP symptoms, however, have not been achieved with most studies. Thereby, alternative therapeutic options for this bothersome condition are needed (12-15).

Aloe vera contains multiple antioxidant compounds which have anti-inflammatory effects including superoxide dismutase, vitamins $C$ and $E$, etc. (18). Previous studies showed that Aloe vera has been widely used to treat various conditions such as burn wound, radiation-related dermatitis, mucositis, esophagitis, acne vulgaris, and psoriasis vulgaris but none of them investigated the effect of this dry and warm weather plant in $\operatorname{ARP}(1,19-21)$.

Although the overall primary and secondary outcomes of Aloe group were better than the placebo group, with exception of diarrhea, RTOG total scores and CRP, all measures of clinical presentation toxicity did not achieve a statistically significant difference with Aloe vera ointment, compared to the placebo arm. Results from this study have extended the findings of earlier researches and indicated that Aloe vera was at least beneficial in alleviating the bothersome symptoms of radiation-induced damages (22). Aloe vera has shown promising effects in prevention of radiation-induced dermatitis in breast cancer patients receiving $\mathrm{RT}$, through its anti-inflammatory and anti-oxidant properties, likewise to the results observed in the present study (23).

Quantitative-CRP is a non-specific, highly sensitive, acute-phase reactant because of the pronounced rise in concentration after tissue injury or inflammation (24). DNA damage in ARP can increase inflammatory responses and rise in CRP levels. In this study, quantitative CRP was measured during the course of RT, as a reflection of systemic inflammation. As the results of the present study illustrated, CRP serum levels had a descending trend after the treatment with Aloe vera, which was significantly lower in in comparison to the placebo group (Fig. 4). 
The main limitation is the small sample size. It should be pointed out that until now, the efficacy of Aloe vera in ARP prevention in rectal cancer has not been evaluated. Thus, this primary evaluation is the first trial of Aloe vera to gain an insight for future studies. Another limitation was the lack of measurement of chemical constituents of Aloe vera extract that possess antioxidant and anti-inflammatory effects.

Although endoscopic evaluation of the rectosigmoid would be advantageous for response assessment, it has yielded inconsistent descriptions of the character of inflammation. Focal erythema, friability, and mechanical damage are commonly noted. This is the first preliminary clinical trial of treatment with Aloe vera and seeks further studies.

\section{Conclusion}

There is limited evidence on whether topical Aloe vera is effective in preventing radiation-induced proctitis in colorectal cancer patients. The results of this preliminary study supports the preventive effects of this herbal preparation in this population of patients. Further sufficiently powered research studies should be conducted to evaluate the effectiveness of currently used and novel therapies for the prevention, minimization and management of radiation-induced proctitis.

\section{Abbreviations}

ARP: Acute radiation proctitis; RTOG: Radiation Therapy Oncology Group; ARP: Acute radiation proctitis; HAD: Hospital Anxiety-Depression Scale; CRP: C - reactive protein; QOL: Quality of life, GEE: generalized estimating equation.

\section{Declarations}

\section{Acknowledgments}

We gratefully acknowledge the Vice Chancellor of Research and Technology affairs of Mazandaran University of Medical Sciences for financial support.

\section{Authors' contributions}

Conception and design: ES, AG, JA and AS. Data collection: AS, and HRG. Data analysis and manuscript preparation: ES, AS, FS, MS, and AA. Final approval of manuscript: All authors.

\section{Funding}


This study was financially supported by a grant from the Research and Technology Department of Mazandaran University of Medical Sciences, Sari, Iran. This grant providing funding to give medical agents, data analysis and editorial assistance with the writing of the manuscript but had no role in study design and data collection.

\section{Availability of data and materials}

All data generated or analyzed during this study are included in this published article.

\section{Ethics approval and consent to participate}

All patients signed an informed consent form prior to participation in the study. The study protocol was approved by the Ethics Committee of Mazandaran University of Medical Sciences (IR.MAZUMS.REC.941196) and registered in the Iranian Registry of Clinical Trials (IRCT201606042027N6).

\section{Consent for publication}

Not applicable.

\section{Competing interests}

The authors declare that they have no relevant competing interests.

\section{References}

1. Richardson J, Smith JE, Mclntyre M, Thomas R, Pilkington K. Aloe vera for preventing radiationinduced skin reactions: a systematic literature review. Clin Oncol. 2005;17(6):478-84.

2. Sahebnasagh A, Ghasemi A, Akbari J, Alipour A, Lashkardoost H, Ala S, et al. Successful treatment of acute radiation proctitis with aloe vera: a preliminary randomized controlled clinical trial. The Journal of Alternative Complementary Medicine. 2017;23(11):858-65.

3. Sahu PK, Giri DD, Singh R, Pandey P, Gupta S, Shrivastava AK, et al. Therapeutic and medicinal uses of Aloe vera: a review. Pharmacology Pharmacy. 2013;4(08):599.

4. Kennedy M, Bruninga K, Mutlu EA, Losurdo J, Choudhary S, Keshavarzian A. Successful and sustained treatment of chronic radiation proctitis with antioxidant vitamins $\mathrm{E}$ and $\mathrm{C}$. Am J Gastroenterol. 2001;96(4):1080-4. 
5. Denton A, Andreyev H, Forbes A, Maher E. Systematic review for non-surgical interventions for the management of late radiation proctitis. British journal of cancer. 2002;87(2):134.

6. Widmark A, Fransson P, Tavelin B. Self-assessment questionnaire for evaluating urinary and intestinal late side effects after pelvic radiotherapy in patients with prostate cancer compared with an age-matched control population. Cancer. 1994;74(9):2520-32.

7. Majumdar SR, Fletcher RH, Evans AT. How does colorectal cancer present? Symptoms, duration, and clues to location. Am J Gastroenterol. 1999;94(10):3039-45.

8. Cappell MS. Pathophysiology, clinical presentation, and management of colon cancer. Gastroenterology Clinics. 2008;37(1):1-24.

9. Colorectal CCG. Adjuvant radiotherapy for rectal cancer: a systematic overview of 8,507 patients from 22 randomised trials. 2001.

10. Johnston M, Robertson G, Frizelle F. Management of late complications of pelvic radiation in the rectum and anus. Diseases of the colon rectum. 2003;46(2):247-59.

11. Lee WR, Hanks GE, Hanlon AL, Schultheiss TE, Hunt MA. Lateral rectal shielding reduces late rectal morbidity following high dose three-dimensional conformal radiation therapy for clinically localized prostate cancer: further evidence for a significant dose effect. International Journal of Radiation Oncology• Biology• Physics. 1996;35(2):251-7.

12. Sarin A, Safar B. Management of radiation proctitis. Gastroenterology Clinics. 2013;42(4):913-25.

13. Staffurth J. A review of the clinical evidence for intensity-modulated radiotherapy. Clinical oncology. 2010;22(8):643-57.

14. Singh AK, Ménard C, Guion P, Simone NL, Smith S, Crouse NS, et al. Intrarectal amifostine suspension may protect against acute proctitis during radiation therapy for prostate cancer: a pilot study. International Journal of Radiation Oncology• Biology• Physics. 2006;65(4):1008-13.

15. Kouloulias VE, Kouvaris JR, Pissakas G, Mallas E, Antypas C, Kokakis JD, et al. Phase II multicenter randomized study of amifostine for prevention of acute radiation rectal toxicity: topical intrarectal versus subcutaneous application. International Journal of Radiation Oncology• Biology• Physics. 2005;62(2):486-93.

16. Najafı M, Motevaseli E, Shirazi A, Geraily G, Rezaeyan A, Norouzi F, et al. Mechanisms of inflammatory responses to radiation and normal tissues toxicity: clinical implications. Int J Radiat Biol. 2018;94(4):335-56.

17. Rafati M, Ghasemi A, Saeedi M, Habibi E, Salehifar E, Mosazadeh M, et al. Nigella sativa L. for Prevention of Acute Radiation Dermatitis in Breast Cancer: A Randomized, Double-blind, Placebocontrolled, Clinical Trial. Complementary Therapies in Medicine. 2019:102205.

18. Ahmadi A. Potential prevention: Aloe vera mouthwash may reduce radiation-induced oral mucositis in head and neck cancer patients. Chin J Integr Med. 2012;18(8):635-40.

19. Paulsen E, Korsholm L, Brandrup F. A double-blind, placebo-controlled study of a commercial Aloe vera gel in the treatment of slight to moderate psoriasis vulgaris. J Eur Acad Dermatol Venereol. 2005;19(3):326-31. 
20. Maenthaisong R, Chaiyakunapruk N, Niruntraporn S, Kongkaew C. The efficacy of aloe vera used for burn wound healing: a systematic review. burns. 2007;33(6):713-8.

21. Hajheydari Z, Saeedi M, Morteza-Semnani K, Soltani A. Effect of Aloe vera topical gel combined with tretinoin in treatment of mild and moderate acne vulgaris: a randomized, double-blind, prospective trial. Journal of Dermatological Treatment. 2014;25(2):123-9.

22. Puataweepong P, Dhanachai M, Dangprasert S, Sithatani C, Sawangsilp T, Narkwong L, et al. The efficacy of oral Aloe vera juice for radiation induced mucositis in head and neck cancer patients: a double-blind placebo-controlled study. Asian Biomed. 2010;3(4):375-82.

23. Di Franco R, Sammarco E, Calvanese MG, De Natale F, Falivene S, DiLecce A, et al. Preventing the acute skin side effects in patients treated with radiotherapy for breast cancer: the use of corneometry in order to evaluate the protective effect of moisturizing creams. Radiat Oncol. 2013;8(1):57.

24. Levinson SS, Elin RJ. What is C-reactive protein telling us about coronary artery disease? Arch Intern Med. 2002;162(4):389-92.

\section{Tables}

Table 1. Baseline Characteristics of Patients

\begin{tabular}{|c|c|c|c|}
\hline Characteristics & Aloe $(n=12)$ & Placebo $(n=8)$ & $P$ value \\
\hline Age, median (IQR), y & $62.5(51-70.5)$ & $49.5(37-61)$ & 0.18 \\
\hline Male sex, No. (\%) & $9(75 \%)$ & $5(62.5 \%)$ & 0.64 \\
\hline BMI, median (IQR), $\mathrm{kg} / \mathrm{m}^{2}$ & $22.4(21-25)$ & $26.6(22-30)$ & 0.06 \\
\hline Dose of radiotherapy in each session, median (IQR), cGy & $180(180-195)$ & $180(180-195)$ & 0.99 \\
\hline Total dose of radiotherapy received, median (IQR), cGy & $5040(5010-5040)$ & $5040(5000-5040)$ & 0.68 \\
\hline
\end{tabular}

BMI, body-mass index

Table 2. Change trends of clinical presentation in Aloe vera and placebo during 6 weeks of follow-up. 


\begin{tabular}{|c|c|c|c|c|c|}
\hline & & \multicolumn{2}{|c|}{ Week } & \multicolumn{2}{|c|}{ Effect } \\
\hline & & 1 & 6 & & \\
\hline & & Median (Q1,Q3)* & Median (Q1,Q3)* & Within group & Between group \\
\hline \multirow[t]{2}{*}{ Rectal Bleeding } & Aloe vera & $1.5(<0.01-2)$ & $<0.01(<0.01-<0.01)$ & $<0.001$ & 0.46 \\
\hline & Placebo & $1(1-1)$ & $1(<0.01-1)$ & 0.24 & \\
\hline \multirow[t]{2}{*}{ Rectal Pain } & Aloe vera & $1(0.25-2)$ & $1(<0.01-1)$ & 0.01 & 0.69 \\
\hline & Placebo & $1(<0.01-1)$ & $1(1-2)$ & 0.5 & \\
\hline \multirow[t]{2}{*}{ Diarrhea } & Aloe vera & $<0.01(<0.01-1)$ & $<0.01(<0.01-<0.01)$ & 0.08 & 0.04 \\
\hline & Placebo & $<0.001(<0.001-1)$ & $1(<0.01-2)$ & 0.69 & \\
\hline \multirow[t]{2}{*}{ Fecal urgency } & Aloe vera & $0.5(<0.01-1.75)$ & $<0.01(<0.01-<0.01)$ & 0.08 & 0.78 \\
\hline & Placebo & $0.5(<0.01-1)$ & $1(<0.01-1)$ & 0.55 & \\
\hline \multirow[t]{2}{*}{ Total Clinical Symptoms } & Aloe vera & $4(0.25-5.75)$ & $1(<0.01-1)$ & $<0.001$ & 0.62 \\
\hline & Placebo & $3(1-4.75)$ & $4(0.5-4)$ & 0.94 & \\
\hline \multirow[t]{2}{*}{ Proctitis } & Aloe vera & $2(<0.01-3)$ & $<0.01(<0.01-<0.01)$ & $<0.001$ & 0.42 \\
\hline & Placebo & $1.5(1-2)$ & $1(1-2)$ & 0.76 & \\
\hline \multirow[t]{2}{*}{ Cystitis } & Aloe vera & $<0.01(<0.01-<0.01)$ & $<0.01(<0.01-1)$ & 0.04 & 0.32 \\
\hline & Placebo & $<0.01(<0.01-0.75)$ & $<0.01(<0.01-1)$ & 0.89 & \\
\hline \multirow[t]{2}{*}{ RTOG Total } & Aloe vera & $3(<0.01-4)$ & $<0.01(<0.01-1)$ & $<0.001$ & 0.02 \\
\hline & Placebo & $2(1-4)$ & $3(2-3)$ & 0.97 & \\
\hline \multirow[t]{2}{*}{ QOL } & Aloe vera & $2(<0.01-2)$ & $<0.01(<0.01-<0.01)$ & $<0.001$ & 0.9 \\
\hline & Placebo & $0.5(<0.01-2)$ & $1(1-2)$ & 0.93 & \\
\hline \multirow[t]{2}{*}{ CRP } & Aloe vera & $2(1-2)$ & $1(1-2)$ & 0.47 & 0.01 \\
\hline & Placebo & $1(1-1)$ & $1.5(1-2)$ & 0.1 & \\
\hline \multirow[t]{2}{*}{ Depression } & Aloe vera & $4(1.25-6.5)$ & $2(<0.01-4)$ & 0.28 & 0.52 \\
\hline & Placebo & $4(2-7.5)$ & $9(4-11)$ & 0.51 & \\
\hline \multirow[t]{2}{*}{ Anxiety } & Aloe vera & $4.5(2.5-6.75)$ & $3(2-3)$ & 0.01 & 0.52 \\
\hline & Placebo & 5 (1.75- 7.75) & $3(2-6)$ & 0.28 & \\
\hline
\end{tabular}




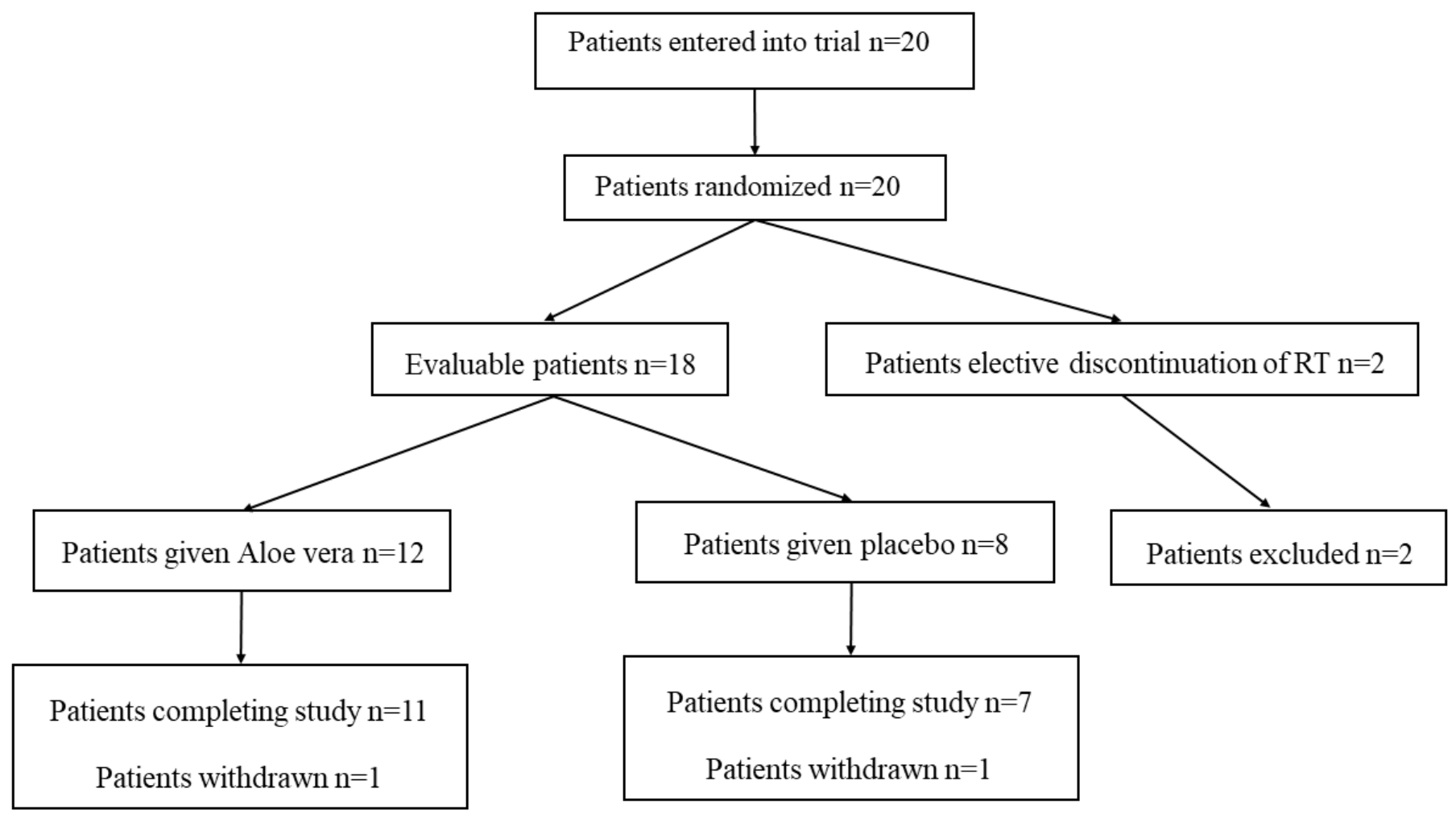

\section{Figure 1}

Flowchart of the study
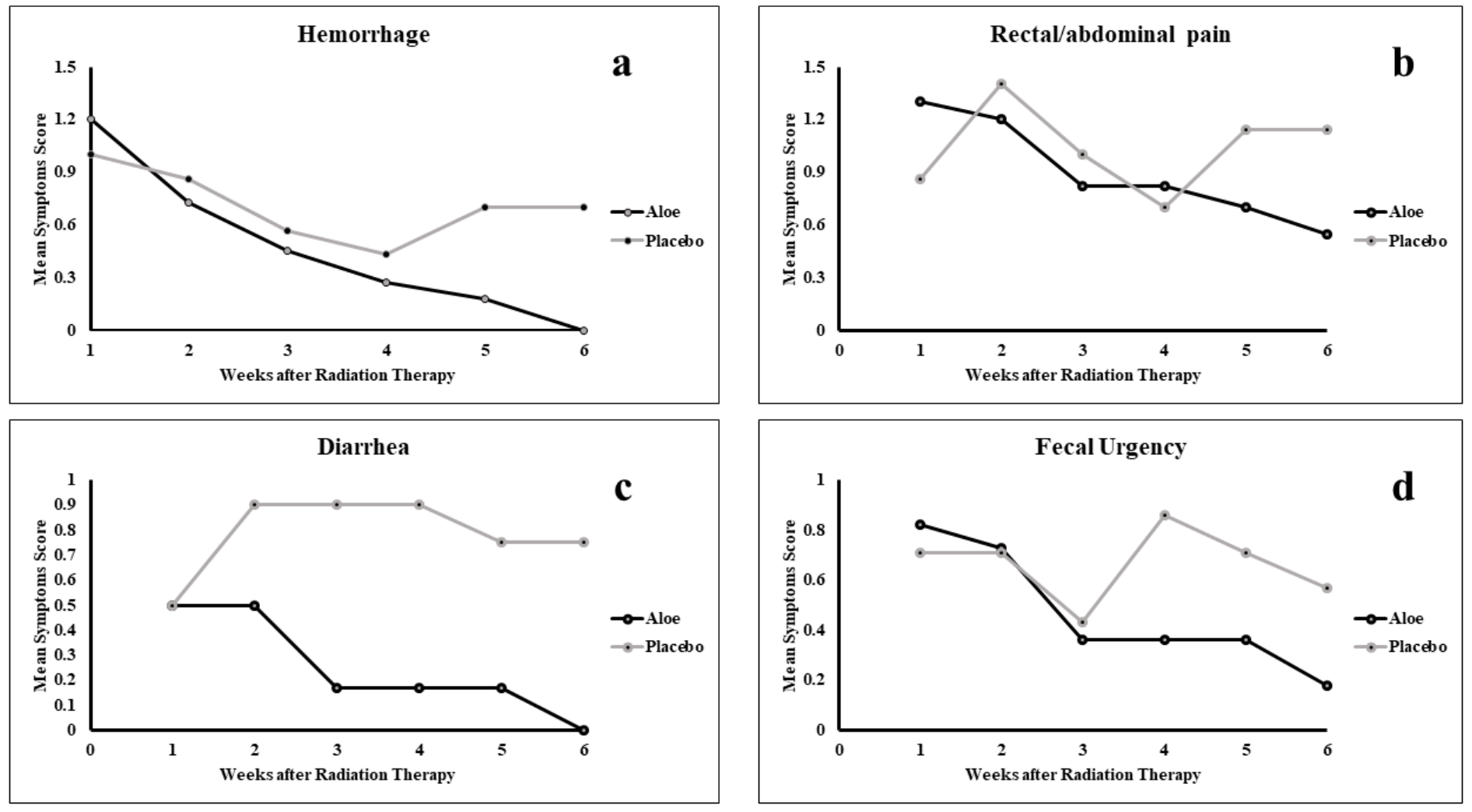
Figure 2

Clinical Symptoms trend of changes during six weeks of follow up: hemorrhage trend of changes (a), abdominal/rectal pain trend of changes (b), diarrhea trend of changes (c), Fecal urgency trend of changes (d).
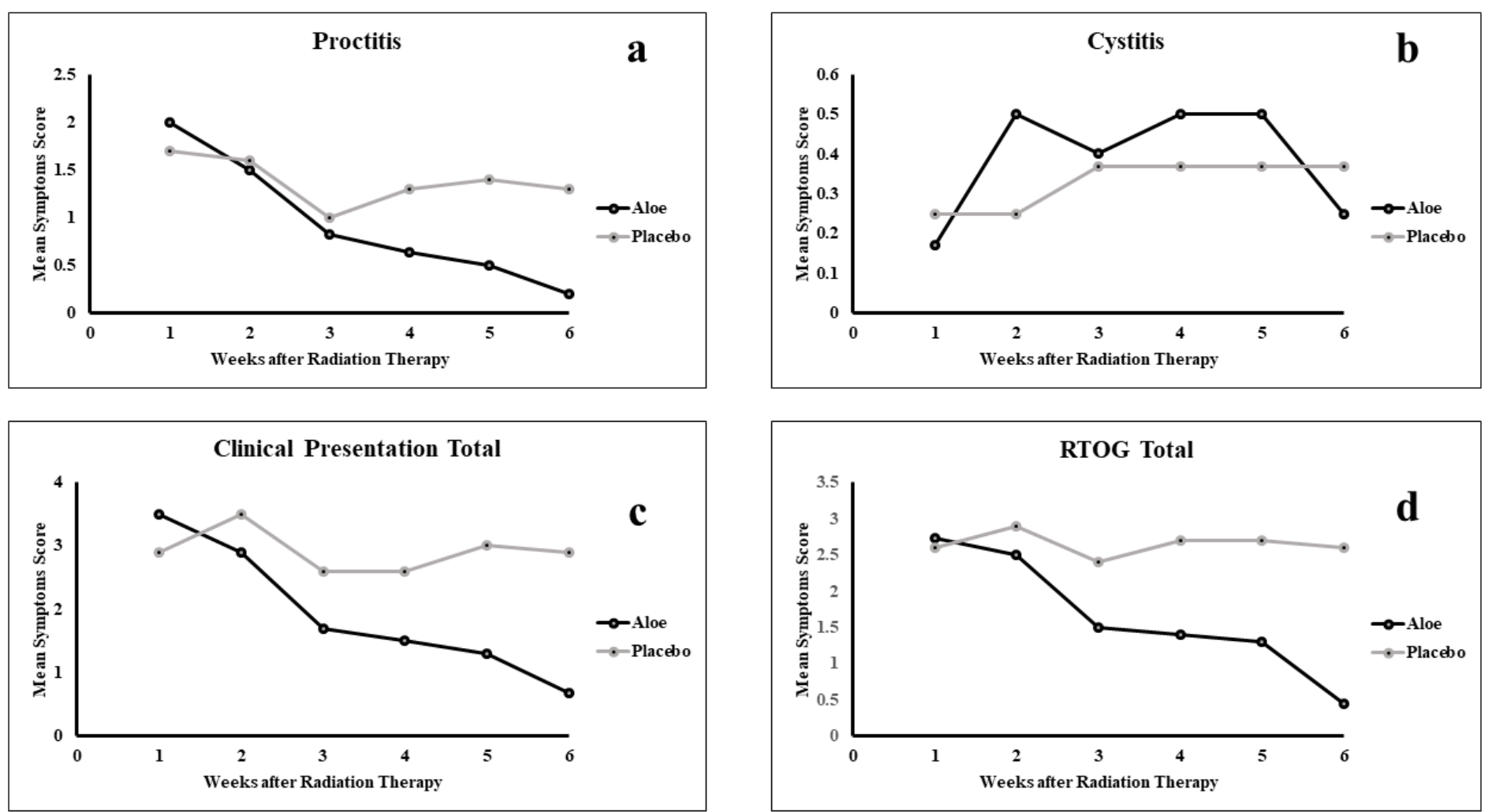

Figure 3

proctitis trend of changes during 6 weeks of follow-up (a), Cystitis trend of changes (b), clinical presentation total trend of changes (c), RTOG total trend of changes (d). 


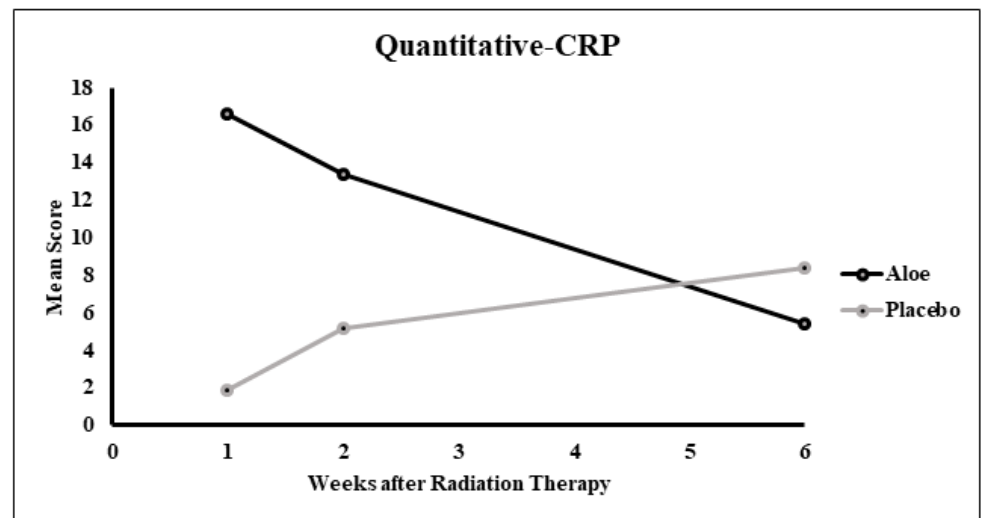

Figure 4

Trends of quantitative CRP levels

\section{Supplementary Files}

This is a list of supplementary files associated with this preprint. Click to download.

- CONSORTChecklist.doc 